\title{
Media and Suicide
}

Thomas Niederkrotenthaler

Prof. Thomas Niederkrotenthaler is associate professor of public health and research group leader for suicide prevention research at Medical University Vienna, Austria. He has worked in several roles within the Association for Suicide Prevention (IASP) in recent years. He has also been involved in revising IASP's funding strategy to emphasize the need to diversify funding to improve and sustain financial and organizational health. Prof. Thomas Niederkrotenthaler has also continuously contributed to the areas he is most interested in, namely communications and media. He has led efforts to develop a communications strategy for IASP activities during Covid-19, has contributed to linking IASP activities with those of the International Suicide Prevention Research Collaboration, and has continuously contributed to briefings on suicide-related media events, for example.

Abstract. Research in the area of media reporting of suicide has a long tradition, and most emphasis has been put exclusively on harmful media potentials (the Werther effect). In recent years, the evidence of increases in suicide subsequent to sensationalist media coverage has substantially increased. Simultaneously, first evidence for the positive roles of portrayals of suicide prevention have emerged as well (the Papageno effect). This presentation will give an overview of recent developments in the field of media and suicide research. The presentation will be a selective review of studies in the field, covering reviews and meta-analytic approaches; content analyses in various media settings; time series analyses; classic pre- after designs to assess associations of media reports with suicide rates, as well as randomized controlled trials conducted with various audiences. There is strong evidence today that media portrayals focusing on suicide methods that are largely non-consistent with media guidelines result in increases in suicidal behaviours, the Werther effect. Strongest evidence comes for celebrity reporting. New evidence has also emerged for selected fictional depictions of suicide. Recent research clearly suggests that both, specific media contents, as well as audience characteristics, play an essential role in suicide-related media effects. Particularly vulnerable individuals who identify with suicidal characters appear most prone to experience media effects, and the type of effect can be an increase or decrease of suicidal ideation, depending on specific me- 
dia contents. Specifically, portrayals of coping with and managing adversity, and featuring help resources, appear to have beneficial effects. Although there are still important gaps in knowledge, the evidence for media effects on suicide and suicidal ideation has clearly increased in recent years. Collaborations with media professionals across media types need to be established, preventive aspects promoted, and these collaborations need to be evaluated. 